\title{
A gestão do cuidado e seus desafios no Estado de Goiás: registro do território e integração entre os níveis assistenciais, 2013-2014
}

Lígia Vanessa Silva Cruz Duarte, Marta Rovery de Souza, Claci Fátima Weirich Rosso

\section{RESUMO}

Motivação: Estudos enfatizam que ações de gestão do cuidado possibilitam melhorias na continuidade do cuidado, na medida em que reduzem barreiras. 0 presente estudo teve como objetivo avaliar a gestão do cuidado na APS utilizando os parâmetros avaliados pelo PMAQ - AB no Estado de Goiás.

Método: Estudo transversal que avaliou os resultados do PMAQ-AB, em 20132014. Foram utilizados dados secundários extraídos do banco de dados da Avaliação Externa do PMAQ-AB.

Resultados: Os resultados desse estudo permitem afirmar que existe fragilidade no que se refere a um registro consistente do território, padronizações para condutas, programação da agenda, oferta de consultas, a classificação de risco e a definição de fluxos bem definidos do usuário dentro do sistema, nas unidades de saúde na Atenção Básica do estado de Goiás.

Conclusões: Os resultados encontrados poderão subsidiar a reflexão dos gestores quanto à identificação de problemas na coordenação, fluxo e continuidade da atenção.

Palavras-chave: Atenção Básica à Saúde; Avaliação Serviços de Saúde; Coordenação do Cuidado; Gestão do Cuidado; Continuidade do Cuidado.
Revista da Rede APS 2019

Publicada em:

24/07/2019

DOI:10.14295/aps.v1i2.32

Lígia Vanessa Silva Cruz Duarte (Instituto de Patologia Tropical e Saúde Pública da

Universidade Federal de Goiás, Brasil), Marta Rovery de Souza (Departamento de Saúde Coletiva do Instituto de Patologia Tropical e Saúde Pública da Universidade Federal de Goiás, Brasil), Claci Fátima Weirich Rosso (Faculdade de Enfermagem da Universidade Federal de Goiás, Brasil);

Correspondência para: Lígia Vanessa Silva Cruz Duarte, ligiaduarteenf@gmail.co m 


\section{INTRODUÇÃO}

Com a criação do Sistema Único de Saúde (SUS), ocorreram grandes mudanças para o modelo de atenção à saúde, até então, não existentes. De um modelo hospitalocêntrico e de caráter curativo, passou-se a um modelo voltado para as ações de promoção, proteção e recuperação da saúde 1-3.

No Brasil, a Atenção Primária à Saúde (APS) tornou-se uma das prioridades nas diretrizes da Política Nacional de Saúde. Nesse contexto, a Portaria no 2.488/GM, de 21 de outubro de 2011, aprovou a Política Nacional de Atenção Básica (PNAB), estabelecendo a revisão de diretrizes e normas para a organização da $A B S$, para a Estratégia Saúde da Família (ESF) e para o Programa de Agentes Comunitários de Saúde (PACS)4. Essa Portaria também conceitua a APS, caracterizando-a como um conjunto de ações de saúde, no âmbito individual e coletivo, que abrange a promoção e a proteção da saúde, a prevenção de agravos, o diagnóstico, o tratamento, a reabilitação, a redução de danos e a manutenção da saúde, com o objetivo de desenvolver uma atenção integral que impacte a condição de saúde, a autonomia das pessoas e os fatores determinantes e condicionantes da saúde57.

Esse Programa foi instituído pela Portaria de $\mathrm{n}$ ㅇ $1.654 \mathrm{GM} / \mathrm{MS}$, do dia 19 de julho de 2011, e foi produto de um importante processo de negociação e pactuação das três esferas de gestão do SUS 5,11 . Ele é desenvolvido em quatro fases, que se complementam e que configuram um ciclo contínuo da melhoria do acesso e da qualidade da APS e são assim denominadas: adesão e contratualização; desenvolvimento; avaliação externa e recontratualização (MINISTÉRIO DA SAÚDE, 2012).

De acordo com Almeida et al. (2010), na América Latina, as reformas centradas na gestão do cuidado ainda são incipientes, contribuindo para um número limitado de estudos que tratam deste tema na região. Nestas circunstâncias, apesar da APS ser colocada no Brasil como gestora do cuidado na rede de atenção, esse atributo se configura como um desafio permanente para as equipes $7,9,14$. Isto justifica a realização de um maior número de pesquisas que possam contribuir para a compreensão das barreiras que dificultam esse processo, permitindo assim, repensar melhorias e práticas de gestão que contribuam para construir a viabilidade de uma atenção mais contínua, integrada e com melhor qualidade.

Desse modo, considerando as questões colocadas, cabe perguntar: a APS tem cumprido a sua função de coordenadora do cuidado em sistemas municipais de saúde? Quais os principais obstáculos das equipes para cumprir a função de coordenadora do cuidado na APS?

O presente estudo teve como objetivo avaliar a gestão do cuidado no âmbito da APS utilizando os parâmetros avaliados pelo $\mathrm{PMAQ}$ - AB em Goiás.

\section{MÉTODO}

Trata-se de um estudo descritivo de corte transversal aninhado à uma pesquisa nacional realizada pelo $M S$, que avalia a APS em todo País, e está vinculada ao PMAQ - $A B$, cujo objetivo é induzir a ampliação do acesso e a melhoria da qualidade na APS.

O banco de dados disponibilizado pelo MS é organizado em Módulos I, II e III. No presente estudo, foi feita a análise dos dados do Módulo II. Para cada uma das situações, foram feitas estatísticas descritivas utilizando as frequências absolutas e relativas. A análise estatística foi realizada utilizando-se o programa IBM ${ }^{\circledast}$ SPSS $^{\circledR}$ Statistics, versão 23.0.

A presente pesquisa foi aprovada pelo Comitê de Ética em Pesquisa da Universidade Federal de Pelotas, Protocolo no 38/12, e teve como base os cuidados éticos necessários para pesquisa com seres humanos preconizados pela Resolução CNS no 466, de 12 de dezembro de 2012. 


\section{Resultados}

Nesse estudo, a amostra foi composta por 1.180 UBS integrantes da ABS no Estado de Goiás. Essas UBS foram incluídas no 2 o ciclo da Pesquisa Nacional de Avaliação realizada pelo MS, por meio do PMAQ-AB.

Um ponto importante para a gestão do cuidado é o registro da população considerada vulnerável. Os públicos avaliados para registro foram os seguintes: gestantes, mulheres elegíveis para exame citopatológico de colo do útero, mulheres elegíveis para mamografia, crianças até dois anos, pessoas com hipertensão, diabetes, com DPOC/Asma, obesidade e outros (Figura 1).

Em 1.096 EAP (92,9\%), há registro às gestantes de seu território, 1.028 EAP $(87,1 \%)$ registram os hipertensos, 1.023 EAP (86,7\%) os diabéticos e 914 $\operatorname{EAP}(77,5 \%)$ fazem registro das crianças menores de dois anos (Figura 1).

Percebe-se que apenas 467 EAP $(39,6 \%)$ registram esse grupo, seguidas de $306 \operatorname{EAP}(25,9 \%)$ para os indivíduos obesos e 297 EAP (25,2\%) que registraram os usuários portadores de DPOC (Tabela 1).

Além disso, vale ressaltar que do total de unidades que relataram registrar esses grupos vulneráveis dentro do seu território, um pequeno percentual confirmou essas ações por meio de registros $(13,8 \%)$ (Tabela 1).

Quando se fala em registro dos usuários que foram encaminhados a outro ponto de atenção na rede, os números caem em praticamente todos os grupos estudados. Isso demonstra perda do acompanhamento do usuário entre os níveis assistenciais. Um número considerável de equipes de saúde $454(38,4 \%)$ não registra o fluxo do paciente

Instrumentos de referência e contrarreferência representam são importantes para integração do serviço de saúde. Foi verificado que, na maior parte dos casos, não há um bom ordenamento e definição no fluxo, visto que, uma porcentagem significativa das equipes não tem retorno, em relação aos encaminhamentos (60,9\%) (Figura 1 ).

\section{DISCUSSÃO}

Uma gestão ideal envolve abordagens multidisciplinares, a documentação dos serviços prestados aos usuários, a comunicação, o monitoramento e a integração na prestação de serviços 3,15-20. Segundo esses autores, a gestão do cuidado, dentre outras coisas, se refere a solucionar problemas de comunicação, para compartilhar informações e conhecimentos entre os profissionais envolvidos na assistência.

Estudos apontam que, quando os sistemas prestam cuidados mais coordenados, há minimização de barreiras de acesso entre níveis assistenciais, oferecendo atenção em saúde mais sincronizada e em tempo oportuno21. A APS é o local que deve se responsabilizar por gerir o percurso do cuidado na maior parte dos episódios de adoecimento.

Os principais instrumentos de gestão do cuidado avaliados pelo PMAQ-AB são: busca ativa, registro de encaminhamentos dentro do sistema, protocolos, registro do território e a referência e contrarreferência.

A busca ativa é um instrumento de suma importância no conjunto de ações em vigilância epidemiológica de investigação de campo, e tem como objetivo a identificação precoce de casos suspeitos e rápida confirmação para orientar adequadamente a aplicação de medidas de controle. Contudo, quando se fala em saúde pública, busca ativa passou a ser entendida como um movimento de cartografar as necessidades de saúde da população22,23..

Outra questão que deve ser abordada quando se fala em gestão do cuidado é em relação ao registro do território pelas unidades de saúde. Percebemos, após a análise dos dados, que alguns públicos têm maior registro em todas as porções populacionais, como as gestantes, hipertensos, 
diabéticos e crianças menores de dois anos, enquanto grupos como obesos, mulheres elegíveis para mamografia e usuários com DPOC/asma não têm registro com tanta frequência.

Além disso, vale ressaltar que do total de unidades que relataram registrar esses grupos vulneráveis dentro do seu território, um pequeno percentual confirmou essas ações através de registros.

Outro mecanismo de gestão e de redução da variedade das condutas é o uso de protocolos clínicos. A ESF utiliza manuais com protocolos clínicos elaborados pelo MS para acompanhamento dos grupos prioritários, como os protocolos de assistência integrada às doenças prevalecentes na infância, hipertensão arterial, diabetes, planejamento familiar, saúde da mulher, pré-natal, doenças sexualmente transmissíveis e muitos outros 24 . Em Goiás, $61,6 \%$ das EAP referiram não se utilizar de protocolos para nortear suas ações. Um componente essencial da gestão do cuidado se refere à utilização de ferramentas para padronização de condutas, como os protocolos clínicos e as diretrizes baseadas em evidência. Estas medidas teriam como objetivo facilitar os cuidados dos usuários da atenção primária que necessitam de assistência em outros níveis de atenção, e até mesmo, diminuir o número de encaminhamentos desnecessários. Autores ressaltaram a necessidade de práticas norteadas por protocolos e dos impactos destes na gestão do cuidado8,18.

O desenvolvimento de instrumentos de coordenação clínica como protocolos representa estratégia para garantia atenção integral, tendo como o centro os serviços da rede básica. Embora se perceba iniciativa de uso de instrumentos para promover coordenação, alguns desafios permanecem, como a existência de diferentes prestadores de serviço, ausência de mecanismos de referência e contrarreferência, falta de comunicação efetiva entre os níveis de atenção e necessidade de maior integração 25.

A integração das Unidades de Saúde da Família à rede assistencial é fundamental para garantir uma oferta abrangente de serviços, e para gerir as diversas ações requeridas para resolver as necessidades menos frequentes e mais complexas 21. A gestão implica a capacidade de garantir a continuidade da atenção ao longo da rede de serviços, por meio de mecanismos de integração, tecnologias de gestão clínica e instrumentos para a comunicação entre profissionais de diferentes serviços.

A comunicação com troca de informações entre profissionais é essencial para que o generalista da equipe de saúde da família possa exercer sua função de coordenador dos cuidados ao paciente e garantir a continuidade do contato. Embora existissem formulários para a transferência de informações entre médicos do PSF e especialistas, na maioria das unidades, seu uso não é frequente $(60,9 \%)$. A insuficiente realização da contrarreferência foi uma dificuldade reiterada pelas unidades na maior parte dos municípios estudados.

Uma gestão ideal envolve abordagens multidisciplinares, a documentação dos serviços prestados aos usuários, a comunicação, o monitoramento e a integração na prestação de serviços 3,15-17. Segundo estes autores, gestão do cuidado se refere a solucionar problemas de comunicação para compartilhar informações e conhecimentos entre os profissionais envolvidos na assistência.

Nas práticas de gestão, estudos indicaram a importância da avaliação dos pacientes para identificar necessidades e demandas assistenciais, identificar populações mais vulneráveis ao cuidado fragmentado e determinar prováveis desafios de gestão 20.

Gerir o cuidado se relacionaria com a gerência de práticas e fluxos de informações de saúde, por meio de mecanismos de referência, com o intuito de efetivar a integração dos serviços de atenção primária com serviços especializados 26 .

Quanto às evidências sobre mecanismos de gestão do cuidado, um estudo destacou a referência e a contrarreferência como um meio para viabilizar o encaminhamento de usuários para especialistas e 
Vol. 1, n. 2, p. 121/131 | Maio/Agosto - 2019

ISSN 2596-3317 - DOI 10.14295/aps.v1i2.32

Duarte, L. V. S. C.; Souza, M. R.; Rosso, C. F. W.

atenção hospitalar. No entanto, a contrarreferência se mostrou como a prática pouco comum, apesar de ter sido apontada como prática fundamental para assegurar o acompanhamento do paciente 18 .

A existência de mecanismos e referência e contrarreferência são importantíssimos para uma melhor comunicação e integração entre a rede. A insuficiência na oferta de atenção especializada, agravada pela pouca integração entre prestadores estaduais e municipais, foi outra dificuldade identificada. A quase ausência de contrarreferência também minimiza as possibilidades de gestão dos cuidados pela equipe de APS.

É necessário criar modalidades de cuidado que correspondam às necessidades do usuário, com um olhar cuidadoso sob o território. A APS é o cenário ideal para se trabalhar a relação entre os serviços, a discussão de casos, as singularidades do território, a escuta dos usuários e a visita domiciliar. A gestão do cuidado contribui para a qualificação do cuidado.

Foi possível observar uma fragilidade no registro consistente do território, na programação da agenda, na oferta de consultas e na classificação de risco.

Percebe-se também uma deficiência nos fluxos de informações de saúde, por meio de mecanismos de referência, com o intuito de efetivar a integração dos serviços de APS com serviços especializados.

Um número significativo de EAP não se orienta por protocolos e diretrizes clínicas, sendo que o MS oferece diversos manuais para facilitar essa prática. Tal conduta gera diversas ações diferentes para um mesmo fim, provavelmente, aumentando os custos e diminuindo a resolubilidade.

Desse modo, essa análise, na perspectiva da gestão, visa direcionar ou redirecionar as prioridades municipais, permitindo o correto investimento no fortalecimento da ABS.

Os resultados encontrados poderão subsidiar a reflexão dos gestores quanto à identificação de problemas na coordenação, ao fluxo e continuidade da atenção, e auxiliar no planejamento, na programação e na avaliação de medidas de intervenção a serem adotadas pela Secretaria do Estado da Saúde de Goiás e pelas Secretarias Municipais de Saúde, a fim de estruturar a APS, com o intuito de torná-la mais resolutiva e menos dispendiosa.

Isso justifica a realização de um mais pesquisas que possam contribuir para a compreensão das barreiras que dificultam esse processo, permitindo assim, repensar melhorias e práticas de gestão pública que contribuam para a viabilidade de uma atenção à saúde mais contínua, integrada e com melhor qualidade em Goiás.

\section{REFERÊNCIAS BIBLIOGRÁFICAS:}

(1) Brasil. Constituição da República Federativa do Brasil. Brasília: Senado Federal, 1988. 486 p. Disponível em: https://www2.senado.leg.br/bdsf/bitstream/handle /id/508200/CF88_EC85.pdf. Acessado em: 01 set. 2018.

(2) Valentim IVL, Kruel AJ. A importância da confiança interpessoal para a consolidação do Programa de Saúde da Família. Ciênc. Saúde Colet. 2007; 12(3):777-88.

(3). Ministério da Saúde. Portaria no 699, de 22 de fevereiro de 2006. Divulga o Pacto pela Saúde 2006 - Consolidação do SUS e aprova as Diretrizes Operacionais do Referido Pacto. Brasília: Ministério da Saúde, 2006. Disponível em: http://bvsms.saude.gov.br/bvs/saudelegis/gm/200 6/prt0399_22_02_2006.html. Acessado em: 31 out. 2018.

(4). Ministério da Saúde. Portaria 2.488, de 21 de outubro de 2011. Aprova a Política Nacional de Atenção Básica, estabelecendo a revisão de diretrizes e normas para a organização da Atenção Básica, para a Estratégia Saúde da Família (ESF) e o Programa de Agentes Comunitários de Saúde (PACS). Brasília: Ministério da Saúde, 2012. Disponível em: 
Vol. 1, n. 2, p. 121/131 | Maio/Agosto - 2019

ISSN 2596-3317 - DOI 10.14295/aps.v1i2.32

Duarte, L. V. S. C.; Souza, M. R.; Rosso, C. F. W.

http://bvsms.saude.gov.br/bvs/saudelegis/gm/201 1/prt2488_21_10_2011.html. Acessado em: 25 ago. 2018.

(5). Ministério da Saúde. IDSUS - Índice de Desempenho do Sistema Único de Saúde. Brasília: Ministério da Saúde, 2011. Disponível em: http://idsus.saude.gov.br/. Acessado em: 29 out. 2018.

(6). Ministério da Saúde. Secretaria de Atenção à Saúde. Núcleo Técnico da Política Nacional de Humanização. Ambiência. 2. ed. Brasília: Ministério da Saúde, 2010. 32 p. Disponível em: http://bvsms.saude.gov.br/bvs/publicacoes/ambien cia_2ed.pdf. Acessado em: 25 out. 2018.

(7). Brown R, Schore J, Archibald N, Chen A, Peikes $D$, Sautter $K$, et al. Coordinating care for medicare beneficiaries: early experiences of 15 demonstration programs, their patients, and providers. Mathematica Policy Research, Inc., 2004. 144 p. Disponível em: https://innovation.cms.gov/files/reports/best-praccongressional-report.pdf. Acessado em: 20 set. 2018.

(8). Mcdonald KM, Sundaram V, Bravata DM, Lewis $\mathrm{R}$, Lin $\mathrm{N}$, Kraft $S A$, et al. Care coordination. In: Closing the quality gap: a critical analysis of quality improvement strategies. AHRQ Publication. 2007; 4(7):51-7.

(9). Gittell JH. Organizing work to support relational co-ordination. Int J Human Resource Management. 2000; 11(3):517-39.

(10). Cecílio LCO. A morte de Ivan Ilitch, de Leon Tolstói: elementos para se pensar as múltiplas dimensões da gestão do cuidado. Interface Comunicação, Saúde, Educação. 2009; 13(Supl 1):545-55.

(11). Ministério da Saúde. Portaria no 1.654, de 19 de julho de 2011. Institui no âmbito do Sistema Único de Saúde, o Programa Nacional de Melhoria do Acesso e da Qualidade da Atenção Básica (PMAQ-AB) e o Incentivo Financeiro do PMAQ-AB. Brasília: Ministério da Saúde, 2011. Disponível em: http://bvsms.saude.gov.br/bvs/saudelegis/gm/201 1/prt1654_19_07_2011.html. Acessado em: 23 set. 2018.

(12). Pereira RL, Jeziorsk LL, Fachinni LA. Programa Nacional de Melhoria do Acesso e da Qualidade da Atenção Básica-PMAQ. In: Anais do 21을 Congresso de Iniciação Científica da Universidade de Pelotas, 2012.

(13). Almeida PF, Giovanella L, Mendonça MHM, Escorel S. Desafios à coordenação dos cuidados em saúde: estratégias de integração entre níveis assistenciais em grandes centros urbanos. Cad. Saúde Pública. 2010; 26(2):286-98.

(14). Norris SL, Nichols PJ, Caspersen CJ, Glasgow $R E$, Engelgau MM, Jack $L$, et al. The effectiveness of disease and case management for people with diabetes. A systematic review. Am J Prev Med. 2002; 22 (Suppl 4):15-38.

(15). Gilbert M, Counsell CM. Coordinated care for he SCI patient. SCI Nurs. 1995; 12(3):87-9.

(16). Richards S, Coast J. Interventions to improve access to health and social care after discharge from hospital: a systematic review. J Health Serv Res Policy. 2003; 8(3):171-9.

(17). Boerma WGW. Coordenação e integração em atenção primária europeia. In: Atenção primária conduzindo as redes de atenção. Geneve: WHO, 2006, 344 p.

(18). Hofmarcher MM, Oxley H, Rusticelli E. Improved health system performance through better care coordination. OECD Health Working Papers, n.30, OECD Publishing, 2007.

(19). Pires D. Divisão social do trabalho. In: Escola Politécnica de Saúde Joaquim Venâncio, org. Dicionário da Educação Profissional em Saúde. Rio de Janeiro: Fiocruz, 2009. p.125-30.

(20). Mendes EV. As redes de atenção à saúde. 2. ed. Brasília: Organização Pan-Americana da Saúde, 2011. 549 p. Disponível em: https://www.paho.org/bra/index.php?option=com_ docman\&view=download\&category_slug=servicossaude-095\&alias=1402-as-redes-atencao-a-saude- 


\section{APS em Revista}

Vol. 1, n. 2, p. 121/131 | Maio/Agosto - 2019

ISSN 2596-3317 - DOI 10.14295/aps.v1i2.32

Duarte, L. V. S. C.; Souza, M. R.; Rosso, C. F. W.

2a-edicao-2\&Itemid=965. Acessado em: 3 dez. 2018.

(21). Starfield B. Responsabilidades na atenção primária. In: Atenção primária: equilíbrio entre necessidades de saúde, serviços e tecnologia. Brasília: UNESCO, Ministério da Saúde, 2002. p. 207415. Disponível em: https://www.nescon.medicina.ufmg.br/biblioteca/i magem/0253.pdf. Acessado em: 22 jul. 2018.

(22). Brasil. Ministério da Saúde. Secretaria de Vigilância em Saúde. Guia de Vigilância Epidemiológica. 6. ed. Brasília: Ministério da Saúde, 2005. 816 p. Disponível em: http://bvsms.saude.gov.br/bvs/publicacoes/Guia_V ig_Epid_novo2.pdf. Acessado em 26 ago. 2018.

(23). Souza HM. Saúde da Família: desafios e conquistas. In: Negri B, Viana ALA, org. O Sistema Único de Saúde em dez anos de desafio. São Paulo: Sociedade Brasileira de Vigilância de Medicamentos, Centro de Estudos Augusto Leopoldo Ayrosa Galvão, 2002. p.221-40.

(24). Giovanella L, Escorel S, Mendonça MHM. Porta de entrada pela Atenção Básica? Integração do PSF à rede de serviços de saúde. Saúde Debate,. 2003; 27(65):278-89.

(25). Conill EM, Fausto MCR, Giovanella L. Contribuições da análise comparada para um marco abrangente na avaliação de sistemas orientados pela atenção primária na América Latina. Rev. Bras. Saude Mater. Infant. 2010; 10(Suppl 1):s14-s27.

(26). Santos AM, Giovanella L. Governança regional: estratégias e disputas para gestão em saúde. Rev. Saúde Públ. 2014; 48(4):622-31. 


\section{ARTIGOS}

\section{APS em Revista}

Vol. 1, n. 2, p. 121/131 | Maio/Agosto - 2019

ISSN 2596-3317 - DOI 10.14295/aps.v1i2.32

Duarte, L. V. S. C.; Souza, M. R.; Rosso, C. F. W.

\section{ABSTRACT}

Background: Studies emphasize that care management actions enable improvements in continuity and completeness of care as they reduce barriers. The present study aimed to evaluate the management of care in Primary Health Care using the parameters evaluated by the PMAQ - AB in the State of Goiás.

Methods: This is a cross-sectional study that evaluated the results of the PMAQ-AB, in Goiás, in 2013-2014. Secondary data extracted from the PMAQ-AB External Evaluation database were used.

Results: The results of this study allow us to affirm that there is fragility regarding a consistent registration of the territory, the use of standardizations for conduits, scheduling of the agenda, supply of consultations, risk classification and definition of well defined user flows within the system, in health units in the State of Goiás.

Conclusions: The results found may support the managers' reflection on the identification of coordination problems, flow and continuity of care.

Keywords: The results found may support the managers' reflection on the identification of coordination problems, flow and continuity of care. 


\section{APS em Revista}

Vol. 1, n. 2, p. 121/131 | Maio/Agosto - 2019

ISSN 2596-3317 - DOI 10.14295/aps.v1i2.32

Duarte, L. V. S. C.; Souza, M. R.; Rosso, C. F. W.

Tabela 1. Distribuição percentual de Unidades Básicas de Saúde que realizam registro do território e dos usuários de maior risco encaminhados para outros pontos de atenção, de acordo com a especialidade, em Goiás, 2013-2014

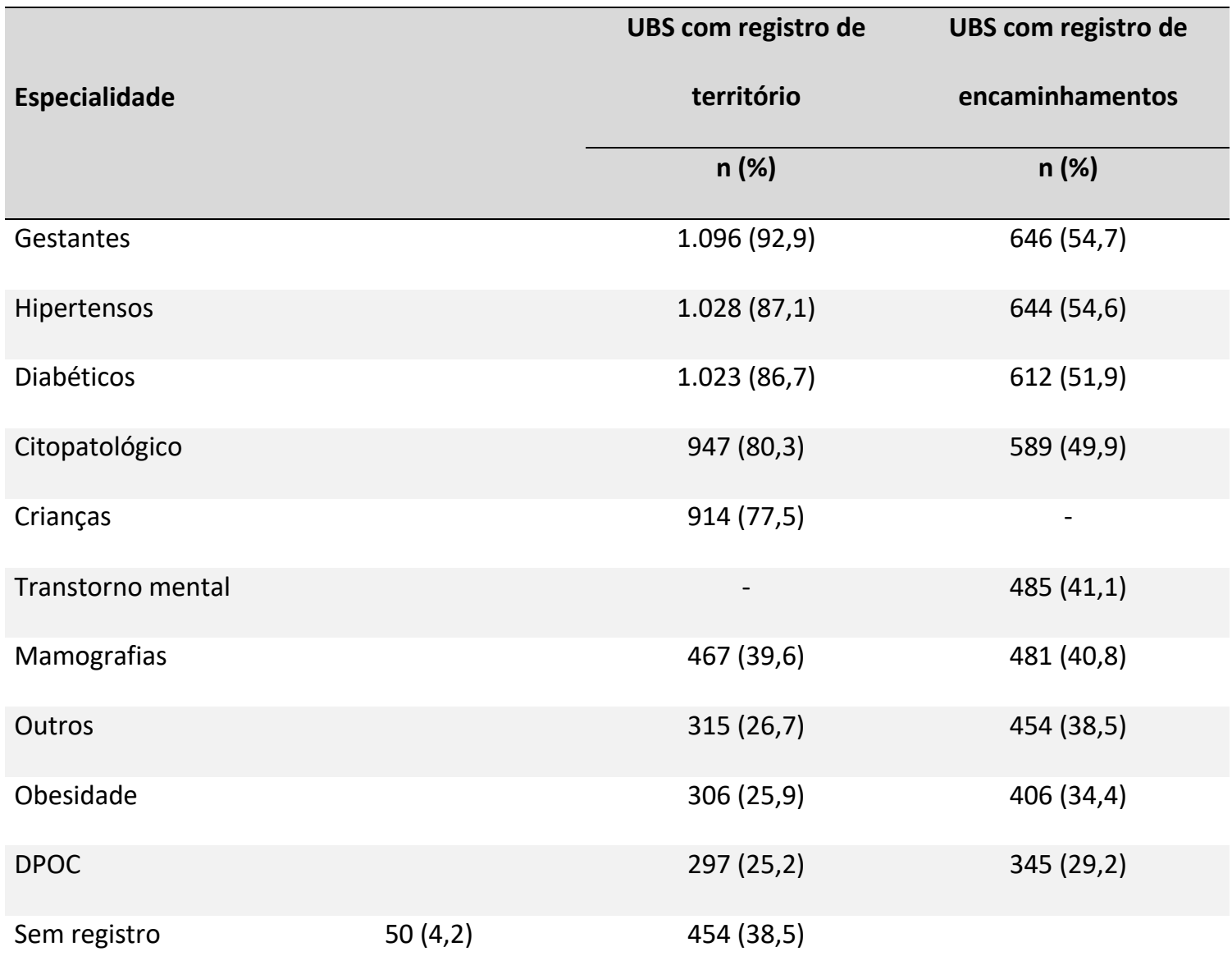

Fonte: Elaboração própria 


\section{APS em Revista}

Vol. 1, n. 2, p. 121/131 | Maio/Agosto - 2019

ISSN 2596-3317 - DOI 10.14295/aps.v1i2.32

Duarte, L. V. S. C.; Souza, M. R.; Rosso, C. F. W.

Figura 1. Distribuição percentual de Unidades Básicas de Saúde que realizam busca ativa, usam protocolos clínicos para classificação de risco e realizam contrarreferência, em Goiás, 2013-2014

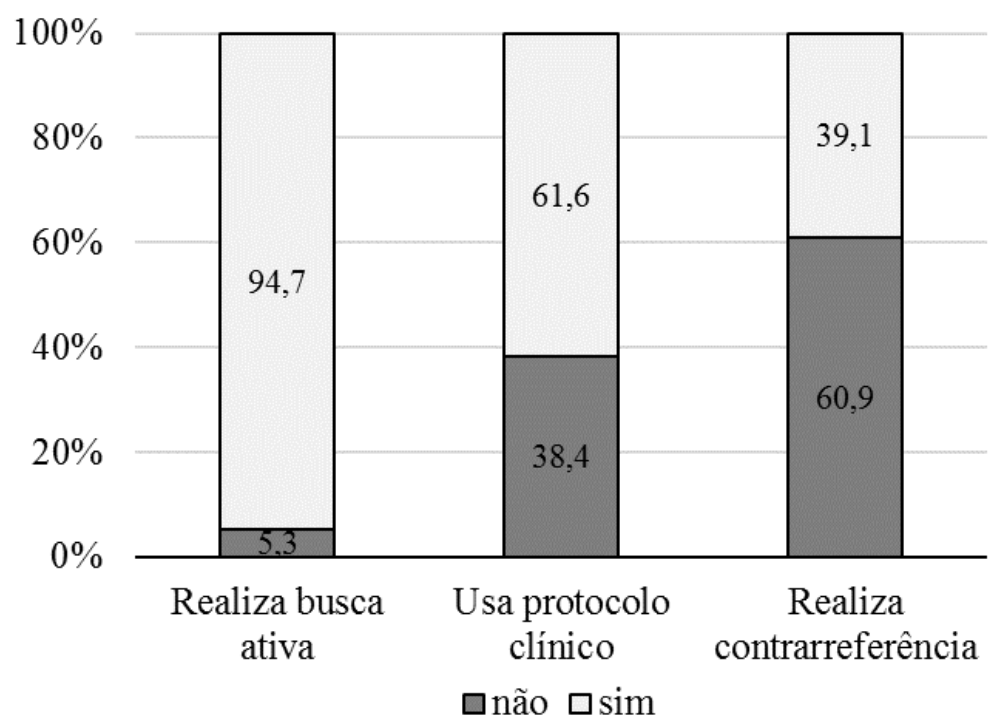

Fonte: Elaboração própria 


\section{APS em Revista}

Vol. 1, n. 2, p. 121/131 | Maio/Agosto - 2019

ISSN 2596-3317-DOI 10.14295/aps.v1i2.32

Duarte, L. V. S. C.; Souza, M. R.; Rosso, C. F. W.

Tabela 2. Distribuição percentual de Unidades Básicas de Saúde que realizam busca ativa e usam protocolo clínico, de acordo com a especialidade, em Goiás, 2013-2014

\begin{tabular}{|c|c|c|}
\hline Especialidade & Realiza busca ativa & $\begin{array}{c}\text { Usa protocolo clínico para } \\
\text { classificação de risco }\end{array}$ \\
\hline & n (\%) & n (\%) \\
\hline Álcool e drogas & $360(30,5)$ & $274(23,2)$ \\
\hline Baixo peso & $838(71,0)$ & - \\
\hline Câncer de mama & $758(64,2)$ & $553(46,9)$ \\
\hline Câncer de útero & $929(78,7)$ & $606(51,4)$ \\
\hline Citopatológico atrasado & $816(69,2)$ & - \\
\hline Crianças & $892(75,6)$ & $587(49,7)$ \\
\hline Diabéticos & $930(78,8)$ & $588(49,8)$ \\
\hline Hanseníase & $927(78,6)$ & $627(53,1)$ \\
\hline Hipertensos & $918(77,8)$ & $587(49,7)$ \\
\hline Pré-natal & $1.025(86,9)$ & $645(54,7)$ \\
\hline Crianças prematuras & $715(60,6)$ & - \\
\hline Puericultura atrasada & $765(64,8)$ & - \\
\hline Saúde mental & $525(44,5)$ & $405(34,3)$ \\
\hline Tuberculose & $887(75,2)$ & $633(53,6)$ \\
\hline Vacinas atrasadas & $947(80,3)$ & - \\
\hline Comprovação & $606(51,4)$ & $616(52,2)$ \\
\hline Doença pulmonar obstrutiva crônica & - & $309(26,2)$ \\
\hline Outros & - & $311(26,4)$ \\
\hline
\end{tabular}

Fonte: Elaboração própria 